\title{
The Adaptive Algorithm Receiving Multipath Signals in the High Frequency Communication Channel Based on the Estimation of its Impulse Response
}

\author{
Aleksandr V. Korennoi, \\ Aleksandr M. Mezhuev and Vadim S. Revin* \\ Military Education and Research Centre of Military-Air Forces \\ «Military-Air Academy \\ Named After Professor N.E. Zhukovsky and Yu.A. Gagarin» \\ 54a Starykh Bolshevikov Str., Voronezh, 394064, Russia
}

Received 12.09.2016, received in revised form 19.11.2016, accepted 07.02.2017

The article deals with an adaptive algorithm for the reception of multipath signals in the high frequency communication channel, built on the basis of experimental data on quasi-stationary channel period. The algorithm allows high-quality offset delayed relative to the direct beam multipath components by providing with accurate synchronization processing between the transmitting and receiving parties, as well as the use of estimates quadrature components of the channel impulse response obtained by the channel sensing signal known shape.

Keywords: adaptive algorithm, multipath, delay time, impulse response.

Citation: Korennoi A.V., Mezhuev A.M., Revin V.S. The adaptive algorithm receiving multipath signals in the high frequency communication channel based on the estimation of its impulse response, J. Sib. Fed. Univ. Eng. technol., 2017, 10(2), 200-210. DOI: 10.17516/1999-494X-2017-10-2-200-210.

(C) Siberian Federal University. All rights reserved

* Corresponding author E-mail address: multitenzor@mail.ru, vadimrevin@bk.ru 


\title{
Адаптивный алгоритм приема многолучевых сигналов \\ в декаметровом канале связи
}

на основе оценки его импульсной характеристики

\author{
А.В. Коренной, А.М. Межуев, В.С. Ревин \\ Военный учебно-научный цеентр Военно-воздуиных сил \\ «Военно-воздушная академия \\ имени профессора Н.Е. Жуковского и Ю.А. Гагарина» \\ Россия, 394064, Воронеж, ул. Старых Большевиков, 54а
}

В статье рассмотрен адаптивный алгоритм приема многолучевых сигналов в декаметровом канале связи, построенный на основе экспериментальных данных о периоде квазистационарности канала. Алгоритм позволяет качественно компенсировать задержаннье относительно прямого луча компоненты многолучевости за счет обеспечения при обработке точной синхронизации между передающей и приемной сторонами, а также использования оценок квадратурных составляющих импульсной характеристики канала, полученных с помощью зондирования канала сигналом известной формыл.

Ключевые слова: адаптивный алгоритм, многолучевость, время задержки, импульсная характеристика.

\section{Введение}

Специфические географические условия России, среди которых прежде всего выделяются следующие: значительная протяженность территории, наличие отдаленных и трудно осваиваемых регионов, близость к приполярным районам и акваториям Мирового океана, существенно ограничивают возможность применения линий электропроводной, радиорелейной, тропосферной и спутниковой связи. В этих условиях важное значение для решения проблемы обеспечения передачи различных видов информации между удаленными на довольно большие расстояния пунктами имеет декаметровая (ДКМ) радиосвязь.

Такие достоинства ДКМ-радиосвязи, как возможность ведения связи на любые расстояния при минимальных энергетических и эксплуатационных затратах, независимость от факторов мировой экономической и политической обстановки, определяют ее дальнейшее использование и развитие в единой сети электросвязи России.

Вместе с тем одной из основных проблем систем ДКМ-радиосвязи является многолучевое распространение сигналов, возникающее из-за многократных отражений радиоволн от неоднородностей ионосферы. Вследствие этого возникает множество лучей, которые накладываются друг на друга на входе приемного устройства. Если же лучи интерферируют в противофазе, то возможно полное исчезновение принимаемого сигнала. Искажения такого рода получили название замираний.

Известно, что существующие системы ДКМ-радиосвязи работают в условиях ограничений на частотные и энергетические ресурсы, поскольку зафиксированы значения выделенной ширины полосы частот канала и мощности передатчика. При этом в силу непредсказуемости условий распространения радиоволн от сеанса к сеансу связи изменяется значение отношения сигнал/шум (ОСШ) и в конкретном сеансе связи оно заранее неизвестно. В результате су-

$$
-201-
$$


ществующие системы ДКМ-радиосвязи обеспечивают среднее значение вероятности битовой ошибки (Bit Error Rate (BER)) в течение суток примерно 10-2. Перспективные системы ДКМсвязи должны обеспечивать в каждом сеансе связи значение BER примерно $10^{-4}[1,2]$.

Таким образом, возникает противоречие между возможностями существующих систем ДКМ-радиосвязи и предъявляемыми к ним требованиями по помехоустойчивости приема сообщений.

Разрешение такого противоречия возможно на основе применения новых адаптивных систем ДКМ-радиосвязи, реализующих эффективные алгоритмы приема. Подобные системы могут быть построены на основе методов активного анализа рабочего канала связи (КС). Эти методы используют специальную процедуру тестирования канала зондирующим сигналом с последующей адаптацией приемного устройства к оценкам параметров КС, полученным в результате тестирования. Таким образом, несомненные преимущества ДКМ-радиосвязи и необходимость обеспечения требуемой помехоустойчивости в условиях многолучевости определяют актуальность темы работы.

\section{Постановка задачи}

В отечественной науке наиболее значимые результаты в области приема многолучевых сигналов и повышения помехоустойчивости в системах ДКМ-радиосвязи получили ученые: О.В. Головин, М.П. Долуханов, Л.М. Финк, Ю.Г. Сосулин, Д.Д. Кловский, П.Ф. Поляков, Б.И. Николаев, В.Г. Карташевский, В.А. Березовский. В зарубежной науке в области указанных направлений широко известны работы ученых C.C. Watterson, B. Widrow, J. Lin, S.G. Tzafestas. Результаты этих исследований свидетельствуют о том, что возможности ионосферного КС далеко не исчерпаны - существует большой резерв в повышении его помехоустойчивости, который возможно реализовать путем разработки новых алгоритмов и моделей приема сигналов в условиях многолучевости.

К числу известных способов приема многолучевых сигналов в ДКМ КС относятся: долговременное и краткосрочное прогнозирование рабочих частот, оперативный маневр частотами на основе данных ионосферного зондирования или трассового опробования, применение автоматической регулировки усиления, различных видов разнесенного приема (по времени, по частоте, по направлению приёма, по поляризации в пространстве), внедрение в радиолинии аппаратуры адаптации, использование помехоустойчивых сигналов, расширение спектра частот сигналов, канальный эквалайзинг, OFDM-мультиплексирование с ортогональным частотным разделением каналов, селективное возбуждение характеристических волн в ионосфере. Выявлено, что каждый способ имеет свои особенности, достоинства и недостатки, однако ни один из них в полной мере не удовлетворяет всем требованиям, предъявляемым к приему сигналов в современной ДКМ-радиосвязи. Поэтому поиск новых оптимальных решений повышения помехоустойчивости систем ДКМ-радиосвязи, безусловно, целесообразен.

Целью работы является разработка адаптивного алгоритма приема многолучевых сигналов в ДКМ КС для повышения помехоустойчивости средств и комплексов ДКМ-радиосвязи в соответствии с заданными требованиями.

Анализ работ [3-7] показал, что характер замираний сигнала на входе приемного устройства исследуемого диапазона постоянно изменяется. Поэтому очевидно, что параметры ДКМ 
КС могут оставаться относительно постоянными (квазистационарными) только на непродолжительных интервалах времени. В результате многочисленных экспериментов, проведенных в самых различных условиях (в разное время суток, года, в различной местности и широтах) при протяженности трассы от 1000 до 3000 км, было установлено, что на входе приемника наблюдается сигнал, содержащий линейную сумму преимущественно 2-4 лучей [8], пришедших с максимальным временем задержки относительно прямого луча $\tau_{\max }=1$ мс [9]. При этом период квазистационарности ДКМ КС составляет $t_{s t} \approx 5-15$ мин [10].

С учетом последнего для обеспечения устойчивого и качественного информационного обмена в ДКМ-диапазоне разработан адаптивный алгоритм приема многолучевых сигналов в ДКМ КС. Его основные этапы:

1. Зондирование КС сигналом известной формы.

2. Оценка квадратурных составляющих ИХ КС $\hat{h}(t, \tau)$.

3. Осуществление информационного обмена в течение периода квазистационарности канала с использованием полученной оценки квадратурных составляющих ИХ для компенсации эффекта многолучевости.

Разберем более подробно реализацию выделенных этапов разработанного алгоритма. Для этой цели рассмотрим представленную на рис. 1 типовую структурную схему ДКМ системы связи, которая включает в себя следующие основные элементы: передающее устройство, многолучевый ионосферный ДКМ КС и приемное устройство. Элементы разработанного адаптивного алгоритма приема многолучевых сигналов в ДКМ КС включены в состав передающего (генератор зондирующих сигналов) и особенно приемного (синхронный детектор, блок оценки параметров многолучевости, блок обработки многолучевых сигналов, блок синхронизации) устройств.

Генератор зондирующих сигналов $u(t)$ передающего устройства используется на первом этапе реализации предлагаемого адаптивного алгоритма приема многолучевых сигналов. Источник сообщений $s(t)$ обеспечивает передачу информационного сигнала непосредственно в ходе информационного обмена.

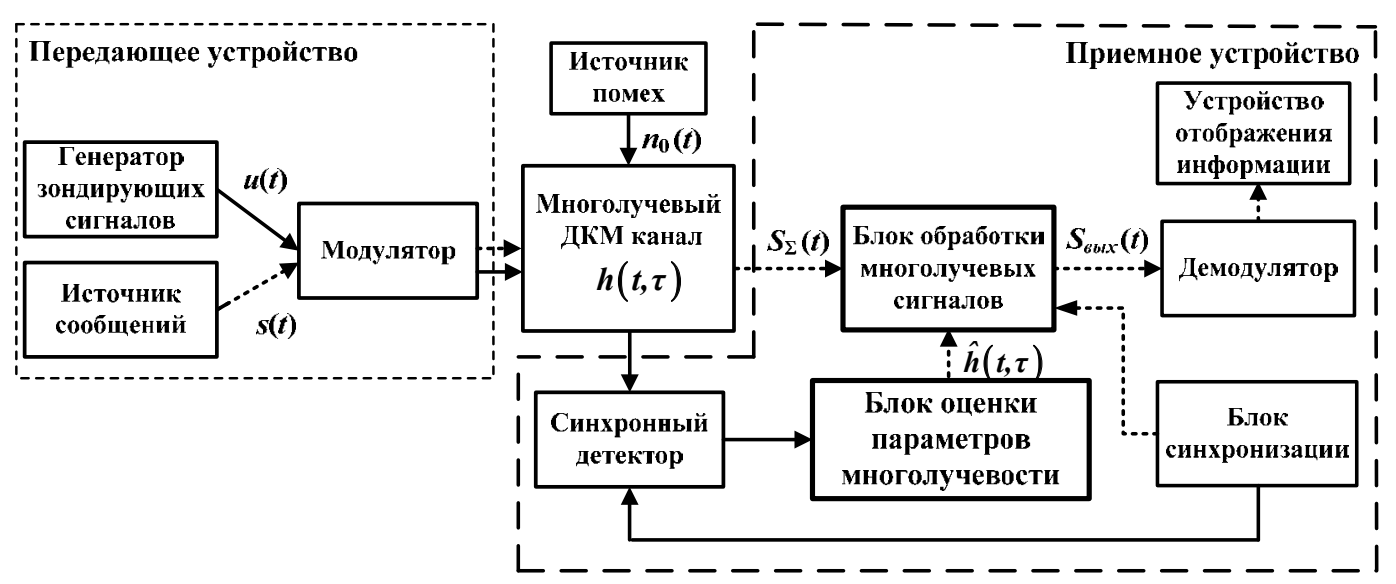

Рис. 1. Упрощенная структурная схема системы ДКМ-радиосвязи с элементами адаптивного алгоритма приема многолучевых сигналов 
При прохождении сигнала через ДКМ КС кроме эффекта многолучевости, вызванного многократными переотражениями от слоев и неоднородностей ионосферы, на него воздействуют различного рода помехи, совокупное влияние которых учитывается аддитивной добавкой к полезному сигналу - шумовой составляющей, представленной известной моделью в виде белого гауссовского шума (БГШ) $n_{0}(t)$.

Приемное устройство, кроме перечисленных выше элементов реализации адаптивного алгоритма приема многолучевых сигналов (синхронный детектор, блок оценки параметров многолучевости, блок обработки многолучевых сигналов, блок синхронизации), включает демодулятор и устройство отображения информации.

Синхронный детектор используется на втором этапе реализации адаптивного алгоритма и с помощью опорного сигнала, синхронизированного с входным сигналом по частоте и фазе (который поступает из блока синхронизации), обеспечивает прямое преобразование реакции ДКМ КС на зондирующий сигнал.

Блок оценки параметров многолучевости обеспечивает реализацию алгоритма фильтрацииинтерполяции квадратурных составляющих ИХ [11], результатом работы которого является получение оценок: количества лучей в принятом многолучевом сигнале, амплитуд лучей и интервалов времени задержки между ними.

Блок обработки многолучевых сигналов обеспечивает компенсацию задержанных компонент многолучевости и выделение прямого луча из суммарного многолучевого сигнала с использованием полученных оценок в блоке оценки параметров многолучевости.

Демодулятор применяют в качестве детектора огибающей, выделяющего НЧ информационный сигнал для передачи его в устройство отображения информации в ходе информационного обмена.

Блок синхронизации обеспечивает жесткую синхронизацию основных преобразовательных, оценочных и компенсационных блоков приемного устройства с передающим, что является основным требованием и особенностью качественной работы схемы приемника при реализации разработанного адаптивного алгоритма.

Распишем более подробно содержание первого этапа предлагаемого адаптивного алгоритма приема многолучевых сигналов в ДКМ КС. На передающей стороне с помощью генератора зондирующих сигналов осуществляется зондирование ДКМ КС сигналом известной формы, в качестве которого используется сигнал с линейно-частотной модуляцией (ЛЧМ). Необходимым требованием к форме зондирующего сигнала является достаточно широкий спектр, равномерно распределенный в полосе частот канала, так как только в этом случае отклик КС на зондирующий сигнал будет с высокой степенью точности совпадать с его ИХ.

ЛЧМ-сигнал с генератора зондирующих сигналов поступает на модулятор, где с помощью высокочастотных колебаний осуществляется его преобразование на заданную несущую частоту диапазона ДКМ волн, на которой в дальнейшем обеспечивается информационный обмен. Сформированный в передающем устройстве высокочастотный зондирующий сигнал излучается передающей антенной в пространство и поступает в ДКМ КС.

Как отмечено выше, при прохождении сигнала через ДКМ КС на него воздействуют различного рода помехи, а вследствие особенностей строения ионосферы он приобретает многолучевый характер из-за многочисленных переотражений от ее слоев и неоднородностей. Реак- 
ция на прохождение зондирующего сигнала через канал в текущий момент времени в виде ИХ ДКМ КС позволяет судить о состоянии ионосферы.

На втором этапе реализации адаптивного алгоритма в приемном устройстве для получения оценки ИХ канала производится устранение высокочастотных составляющих сигнала с использованием синхронного детектора, выполняющего роль приемника прямого преобразования. После синхронного детектора сигнал поступает в блок оценки параметров ИХ КС. В нем на основе квазиоптимального алгоритма [11] осуществляется процедура оценки квадратурных составляющих ИХ ДКМ КС, в результате чего принимается решение о количестве лучей, их амплитуде и времени задержки между ними. Полученные данные используются для компенсации компонент многолучевости в блоке обработки многолучевых сигналов при организации информационного обмена (на третьем этапе реализации алгоритма).

Третий этап осуществляется следующим образом. На передающей стороне информационный сигнал от источника информации поступает в модулятор. Модулятор выполняет те же функции, что и на первом этапе, а именно обеспечивает формирование высокочастотного информационного сигнала для излучения его в КС.

Рассмотрим случай передачи сигнала с двоичной фазовой манипуляцией BPSK, когда одному биту передаваемого сообщения соответствует один символ модулированного гармонического колебания с начальной фазой 0 или $\pi$. Данный вид модуляции нашел широкое применение в ДКМ-системах связи ввиду высокой помехоустойчивости и простоты практической реализации модулятора и демодулятора. В отечественной литературе BPSK-модуляцию обозначают как ФМн-2.

Особенностью третьего этапа предложенного адаптивного алгоритма - в отличие от известных алгоритмов приема многолучевых сигналов в ДКМ КС - является использование полученных на втором этапе оценок квадратурных составляющих ИХ при дальнейшей обработке сигналов в процессе информационного обмена. Для этого в состав схемы включен специально разработанный блок обработки многолучевых сигналов, где в качестве опорных значений по времени $\left(\Delta_{i}\right)$ и весовых коэффициентов по амплитуде $\left(a_{i}\right)$ используются оценки квадратурных составляющих ИХ в виде временных задержек между лучами и их амплитудных значений соответственно (блок оценки параметров многолучевости).

Рассмотрим более подробно функционирование структурной схемы разработанного блока обработки многолучевых сигналов (рис. 2).

Она включает в себя два идентичных канала обработки информационного сигнала. Первый канал предназначен для обработки информационного сигнала «1» с фазой $\pi$, а второй - информационного сигнала «0» с фазой 0 . Работа каналов обеспечивается за счет точной синхронизации по ВЧ заполнению радиоимпульсов BPSK (стрелки «Синхрон.» на рис. 2, показывающие поступление сигналов из блока синхронизации на опорные генераторы $S_{1}(t)$ и $S_{2}(t)$ соответственно верхнего и нижнего плеча).

На входе блока обработки многолучевых сигналов наблюдается аддитивная смесь суммарного многолучевого сигнала $S_{\Sigma}(t)$ и БГШ $n_{0}(t)$

$$
\xi(t)=S_{\Sigma}(t)+n_{0}(t) .
$$

При этом

$$
-205-
$$




$$
S_{\Sigma}(t)=a_{0} S(t)+\sum_{i=1}^{N} a_{i} S\left(t-\Delta_{i}\right),
$$

где $a_{0} S_{1}(t)$ - прямой луч с амплитудой $a_{0} ; a_{i} S\left(t-\Delta_{i}\right)$ - отдельные взаимозадержанные на интервалы времени $\Delta_{i}$ лучи с амплитудами $a_{i} ; i$ - порядковый номер луча; $N$ - общее количество взаимозадержанных лучей в многолучевом сигнале.

Принимаемый сигнал поступает в оба канала обработки. В работе схемы можно выделить два основных этапа:

- компенсация задержанных лучей с использованием параметров, полученных в блоке оценки параметров многолучевости $\hat{h}(t, \tau)$;

- обработка сигнала с использованием согласованной фильтрации (СФ1, СФ2).

Каждый канал включает в свой состав опорный генератор (Оп. ген 1 «1» и Оп. ген 2 «»»), который синхронизирован с передающей стороной для обработки соответственно информационных сигналов «1» и «0».

Опорный генератор Оп. ген $1\left(S_{1}(t)\right)$ генерирует сигналы с фазой, соответствующей информационному сигналу «1», а опорный генератор Оп. ген $2\left(S_{2}(t)\right)$ - с фазой информационного сигнала «0». Эти сигналы используются для синхронизации работы цифровых линий задержки (ЦЛ31 и ЦЛ32 соответственно). ЦЛЗ являются основными элементами блока, обеспечивающими компенсацию задержанных относительно прямого луча, - компонент многолучевости. Для этого в них используются полученные оценки квадратурных составляющих

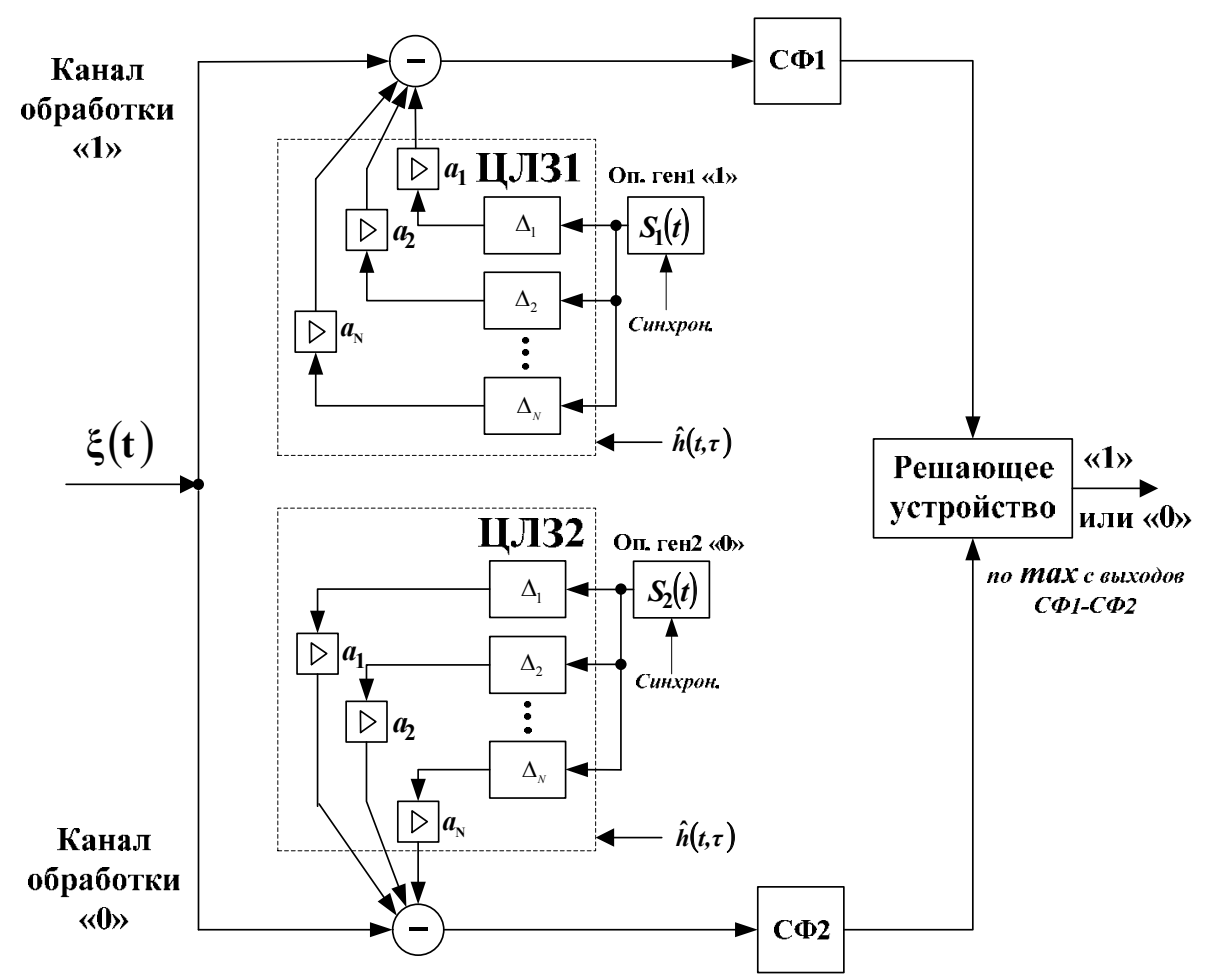

Рис. 2. Структурная схема блока обработки многолучевых сигналов

$$
-206-
$$


ИХ, которые содержат информацию о количестве задержанных лучей, их амплитуде и времени задержки между ними. ЦЛЗ представляет собой перестраиваемый трансверсальный фильтр с $\mathrm{N}$ отводами (в модели устройства $\mathrm{N}$ выбрано равным 10 для максимально возможного случая многолучевого распространения согласно экспериментальным данным). Оценка количества задержанных лучей определяет число используемых отводов ЦЛЗ. Оценки амплитуды компонент многолучевости и временной задержки между ними, соответственно, формируют весовые коэффициенты $a_{i}$ и определяют перестройку времени задержки $\Delta_{i}$ в каждом отводе ЦЛЗ. С помощью сигналов, сформированных на основе оценок ИХ в каждом отводе ЦЛЗ, в вычитающих устройствах каждого канала происходит компенсация компонент многолучевости из аддитивной смеси (2).

Далее сигналы поступают для обработки в согласованные фильтры (СФ) - СФ1 и СФ2. В случае правильной синхронизации по информационному сигналу на первом этапе обработки на выходе СФ наблюдается ярко выраженный максимум ОСШ. В противном случае сигнал на выходе СФ представляет собой результат частичного наложения компонент многолучевости и прямого луча сигнала на входе приемника и ОСШ на выходе фильтра имеет меньшее значение. Полученные в каждом канале блока сигналы поступают на решающее устройство, которое обеспечивает принятие решения о наличии информационного сигнала «1» или «0» по максимальному значению ОСШ, поступающему от СФ.

На заключительном этапе обработки в демодуляторе осуществляется выделение НЧ огибающей информационного сигнала (импульсной информационной последовательности), которая поступает в устройство отображения информации.

\section{Описание основных результатов исследования}

Результаты моделирования обработки сигнала BPSK в многолучевом ДКМ КС представлены на рис. 3. Суммарный многолучевой сигнал (рис. 32) состоит из прямого луча (рис. $3 a$ ) и двух задержанных лучей (рис. 36 и 3в). Если не принимать специальных мер по обработке принятого сигнала, то в результате взаимного наложения лучей друг на друга (эффект многолучевости) сигнал BPSK будет искажен и его правильный прием практически невозможен (рис. 32). В случае использования разработанного адаптивного алгоритма приема многолучевых сигналов (как видно на рис. 3д) передаваемый сигнал может быть восстановлен с высокой точностью и минимальным уровнем искажений.

Для оценки достижения поставленной в работе цели - повышение помехоустойчивости средств и комплексов ДКМ-радиосвязи в условиях многолучевости - были получены зависимости вероятности битовой ошибки BER от значений OCШ (рис. 4).

Кривая 1 построена для случая идеальной синхронизации и отсутствия эффекта многолучевости распространения с использованием классического подхода на основе формулы Крампа. Кривые 2 и 3 являются результатом моделирования в системе Matlab. При этом 2 - кривая помехоустойчивости, полученная при использовании разработанного адаптивного алгоритма приема многолучевых сигналов, 3 - кривая помехоустойчивости без использования блока обработки многолучевых сигналов.

На графиках (рис. 4) видно, что кривая помехоустойчивости 2 при использовании разработанного адаптивного алгоритма приема многолучевых сигналов приближается к идеальному

$$
-207-
$$




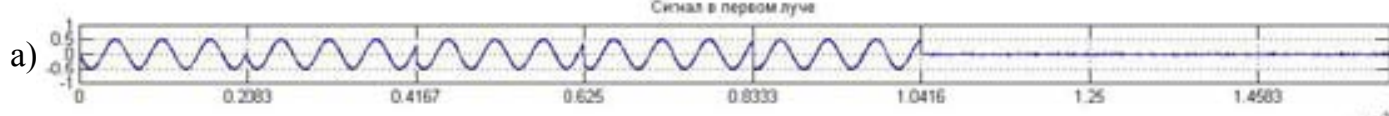
$\times 10^{3}$

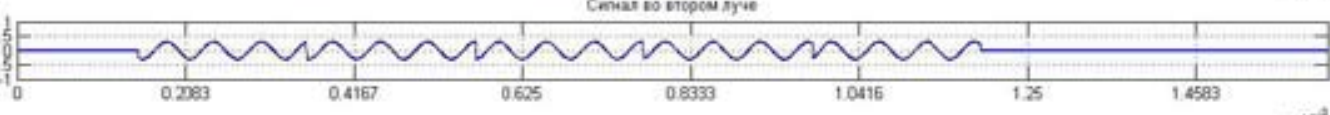

Conan tpeneu spe

B)

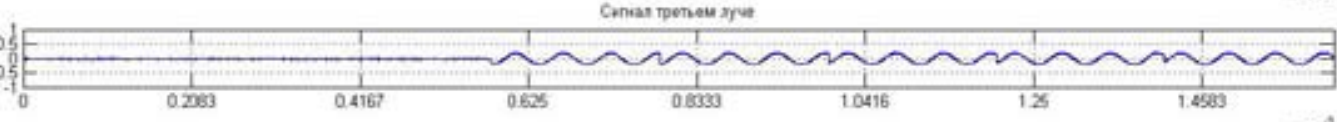

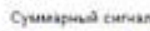

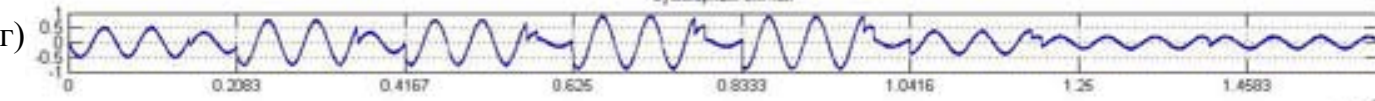

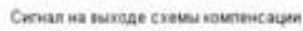

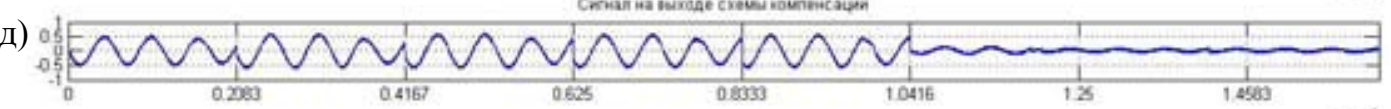

Рис. 3. Результаты моделирования обработки сигнала BPSK при многолучевом распространении в ДКМ КС с использованием разработанного адаптивного алгоритма

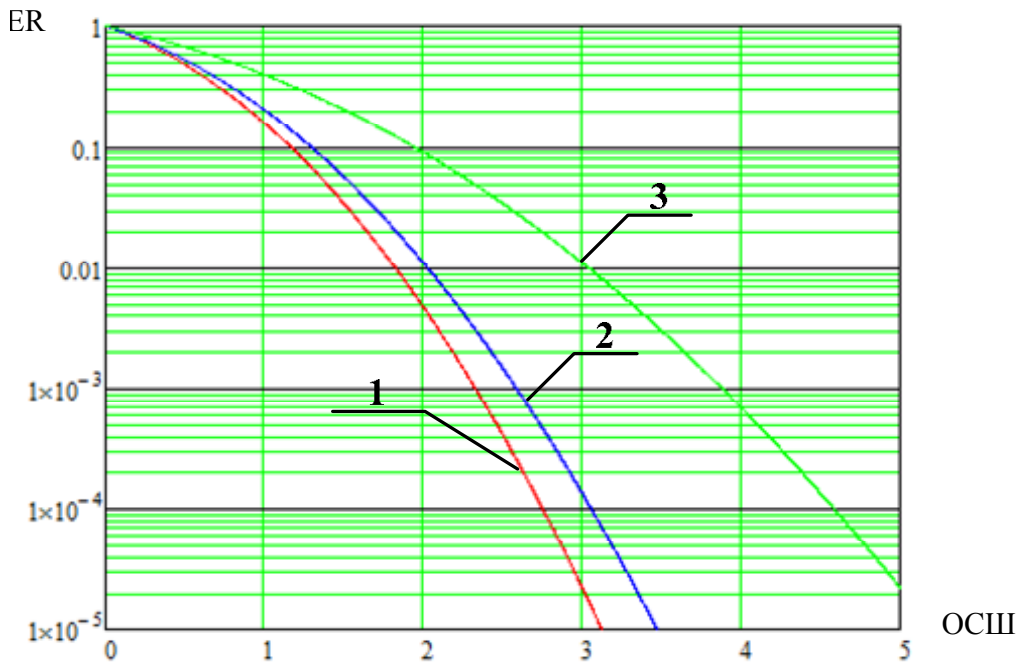

Рис. 4. Кривые помехоустойчивости приема сигналов в ДКМ КС

варианту приема в ДКМ КС без воздействия задержанных лучей (кривая 1). При этом на интересующем уровне вероятности битовой ошибки $\mathrm{BER} \approx 10^{-4}$ отличие в ОСШ составляет всего 1,13 раза. Анализ графиков 2 и 3 показывает, что при одном и том же ОСШ $\approx 3,1$ приемник с предлагаемым блоком обработки многолучевых сигналов обеспечивает требуемую вероятность битовой ошибки для современных комплексов военной ДКМ-радиосвязи (кривая 2), которая на два порядка меньше, чем без использования разработанного блока обработки многолучевых сигналов (кривая 3). В то же время для достижения требуемого значения BER $\approx 10^{-4}$ без использования блока обработки многолучевых сигналов необходимо обеспечить на входе 
приемного устройства ОСШ в 1,5 раза больше, чем в приемнике с предложенным блоком обработки многолучевых сигналов (в 1,64 раза больше по сравнению с идеальным случаем).

\section{Заключение}

На основе экспериментальных данных о периоде квазистационарности ДКМ КС разработан адаптивный алгоритм приема многолучевых сигналов, состоящий из трех основных этапов. Разработана структурная схема системы ДКМ-радиосвязи при использовании предложенного алгоритма. Алгоритм позволяет качественно компенсировать задержанные относительно прямого луча компоненты многолучевости за счет обеспечения при обработке точной синхронизации между передающей и приемной сторонами, а также использования оценок квадратурных составляющих импульсной характеристики канала, полученных с помощью зондирования канала сигналом известной формы.

Проведен анализ работы разработанного адаптивного алгоритма с использованием моделирования функционирования блока обработки многолучевых сигналов при приеме сигналов BPSK, который показал выигрыш на два порядка по значению вероятности битовой ошибки BER (при одном и том же OCШ) по сравнению с типовым приемным устройством, используемым в ДКМ-диапазоне. При этом выигрыш в ОСШ на входе приемного устройства для достижения требуемого значения $\mathrm{BER} \approx 10^{-4}$ составил 1,5 раза.

Таким образом, результаты моделирования свидетельствуют о повышении помехоустойчивости приема многолучевых сигналов в ДКМ КС с использованием разработанного в ходе исследований адаптивного алгоритма и подтверждают достоверность аналитических выкладок и расчетов.

\section{Список литературы}

[1] Землянов И.С. Модемы с ортогональными поднесущими мобильных систем коротковолновой связи с адаптацией к условиям распространения радиоволн. Дис. ... канд. техн. наук: 05.12.04. Омск, 2016. 183 c. [Zemlyanov I.S. Modems with orthogonal podnesem mobile systems high connection with the adaptation to the propagation conditions. Dis. ... PhD in Engineering sciences: 05.12.04. Omsk, 2016, 183 p. (in Russian)].

[2] Котенко О.О. Повымение достоверности передачи информации в радиолиниях коротковолновой радиосвязи на основе применения эффективных сигнально-кодовых конструкций. Дис. ... канд. техн. наук: 05.12.04. СПб., 2013. 165 с. [Kotenko O.O. Improving the reliability of data transmission in the radio links shortwave radio based on the effective signal-to-code designs. Dis.... PhD in Engineering sciences: 05.12.04. Saint Petersburg, 2013. 165 p. (in Russian)].

[3] Агарышев А.И., Михеев С.М. Оперативное прогнозирование оптимальных рабочих частот коротковолнового диапазона для авиационных бортовых комплексов радиосвязи. $\mathrm{Ha}$ учные труды Иркутского ВАИИ, 2001, 3, 6-16 [Agaryshev A.I., Mikheev S.M. Operative prediction of optimal working frequency for aircraft on-Board systems of radio communication. Scientific papers of the Irkutsk MAII, 2001, 3, 6-16 (in Russian)].

[4] Березовский В.А., Фомин В.В., Хазан В.Л. Сеть коротковолновой автоматической радиосвязи для систем мониторинга территориально рассредоточенных объектов. Усnехи современной радиоэлектроники, 2010, 12, 3-16 [Berezovsky V.A., Fomin V.V., Hazan V.L. Automatic

$$
-209-
$$


network shortwave radio for monitoring systems that are geographically dispersed facilities. Successes of modern Radioelectronics, 2010, 12, 3-16 (in Russian)].

[5] Березовский В.А., Дулькейт И.В., Савицкий О.К. Современная декаметровая радиосвязь: оборудование, системы и комплексы. М.: Радиотехника, 2011. 444 с. [Berezovsky V.A., Dulcet I.V., Savitsky O.K. Modern high frequency radio communication: equipment systems and complexes. Moscow, Radio Engineering, 2011. 444 p. (in Russian)].

[6] Лузан Ю.С., Хмырова Н.П. Адаптивная радиосвязь в декаметровом диапазоне частот. Современное состояние и тенденции развития. Техника радиосвязи, 2008, 13, 3-24 [Luzan Y. S., Khmyrov N. P. Adaptive radio in the decameter range of frequencies. Current status and development trends. Radio frequency technician, 2008, 13, 3-24 (in Russian)].

[7] Батенков К.А. Математическое моделирование непрерывных многопараметрических каналов связи в операторной форме. Телекоммуникации, 2013, 10, 2-4 [Batenkov K.A. Mathematical modeling of multivariate continuous communication channels in the operational form. Telecommunications, 2013, 10, 2-4 (in Russian)].

[8] Recommendation ITU-R F.1487 (05/2000). Testing of HF modems with bandwidths of up to about $12 \mathrm{kHz}$ using ionospheric channel simulators. ITU, 2000, 2-13.

[9] Долуханов М.П. Флуктуационные процессы при распространении радиоволн. М.: Связь, 1971. 183 с. [Doluhanov M.P. Fluctuation processes in radio wave propagation. Moscow, Communication, 1971. 183 p. (in Russian)].

[10] Брянцев В.Ф. Способ измерения амплитудно-частотных характеристик ионосферHblx каналов связи. Пат. 2388146, Российская Федерация, 2010, 12, 17 [Bryantsev V.F. Method of measuring amplitude-frequency characteristics of the ionospheric communication channels. Pat. 2388146, Russian Federation, 2010, 12, 17 (in Russian)]

[11] Ревин В.С., Коренной А.В., Межуев А.М. Модель импульсной характеристики декаметрового канала связи и квазиоптимальный алгоритм ее оценивания. Журнал в журнале «Радиосистемы». Формирование и обработка многомерной информации, 2016, 10, 171-176 [Revin V.S., Korennoi A.V., Mezhuev A.M. Model of impulse characteristics of high frequency communication channel and the quasi-optimal algorithm for its evaluation. The journal in the journal «Radio». Acquisition and processing of multidimensional information, 2016, 10, 171-176 (in Russian)]. 